\title{
Leiomyoma of the urinary bladder: a case report
}

\author{
Margarita Ortiz, Daniel E. Henao, Walter Cardona Maya, Maurizio Massaro Ceballos \\ Departament of Radiology (MO, MMC) and Reproduction Group (DEH, CM), University of Antioquia, \\ Colombia
}

\section{ABSTRACT}

The case of a 71-year-old woman who presented with one year history of pelvic pain and occasional dysuria is reported. Computed tomography and Magnetic Resonance Imaging revealed a well defined intramural bladder mass. The histological findings of the surgical specimen confirmed a leiomyoma of the urinary bladder. The clinical presentation, imaging findings and management of this relatively rare benign tumor are discussed.

\section{INTRODUCTION}

Leiomyoma of the bladder is a rare benign tumor that occurs mainly in women between the fourth and fifth decades of life. Although no pathophysiological mechanisms have been described to explain the occurrence of this tumor, it might be related to an endocrine alteration. The most common clinical characteristics include urinary voiding symptoms (1).

We describe in this report the case of an elder woman who mainly presented with chronic pelvic pain. The mass detected within her bladder -by several imaging methods- was later histologically confirmed as leiomyoma.

\section{CASE REPORT}

A 71-year-old female presented with a history of chronic pelvic pain radiating to the low back area associated with occasional dysuria. Poor symptoms relief after several antibiotic treatments for uri- nary tract infections was documented. Initial abdominal ultrasound demonstrated normal kidney aspects and a mass of the bladder (Figure-1).

Subsequent CT of the pelvis confirmed a soft tissue mass at the right anterolateral aspect of the bladder's wall (Figure-2). MR images also demonstrated a mass in the same to location (Figure-3).

The patient underwent a laparoscopic partial cystectomy -after a biopsy of the mass acquired by cystoscopy reported a benign leiomyomaobtaining a surgical specimen of $4 \mathrm{~cm}$ of diameter. Finally, histopathology confirms the diagnosis of leiomyoma (Figure-4).

Figure 1 - Abdominal Ultrasound.

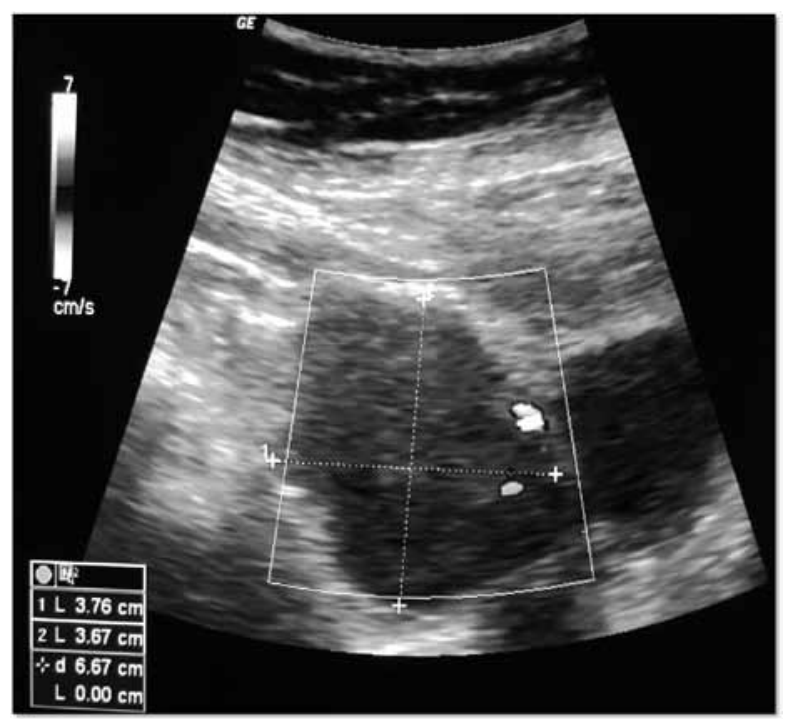

Hypoechoic well-defined mass in the vesical wall measuring $3.67 \mathrm{~cm}$ and $3.76 \mathrm{~cm}$ in their transverse and antero-posterior diameters is shown. According to the Doppler it possesses no blood-flow. 
No recurrence was documented after a year follow-up. The patient experienced a relief of the pain and normal voiding function was retained after surgical excision.

Figure 2 - Computed Tomography (CT) of the pelvis.

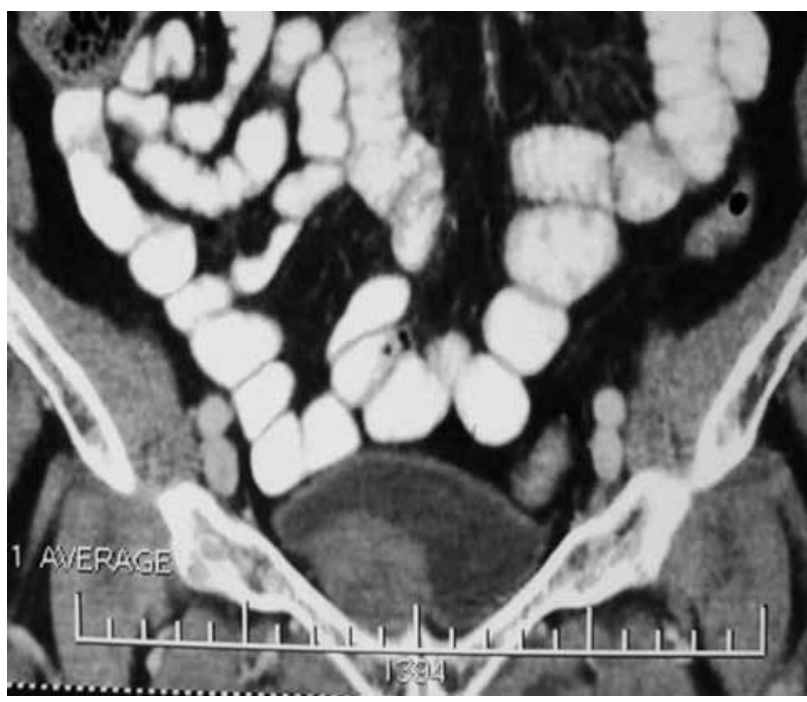

Coronal portal venous phase CT demonstrating a mass with soft tissue attenuation pattern on the right antero-lateral aspect of the bladder's wall is indicated. The mass possesses smooth boundaries with poor enhancement after contrast medium administration demonstrating a homogeneous lesion.

Figure 3 - Magnetic Resonance (MR) Imaging of the pelvis.

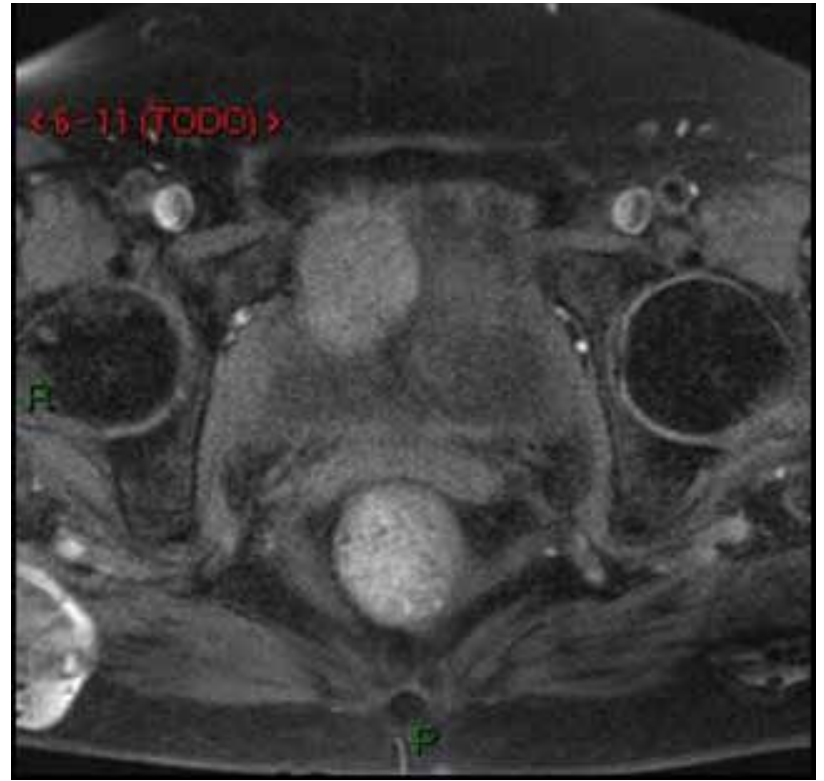

Axial fat-suppressed T1-weighted MR image pre-contrast revealed the mass previously described in the CT scan.
Figure 4 - Piece to specimen.

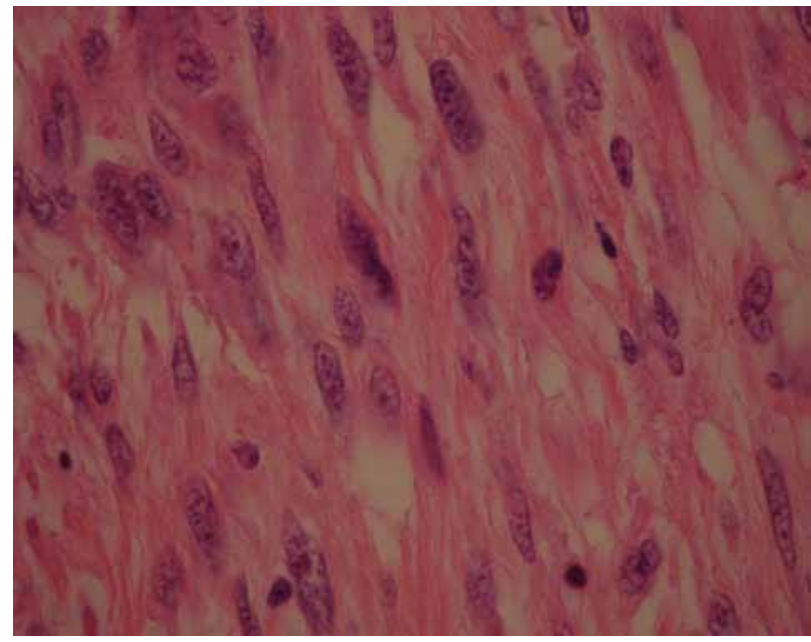

Histopathology of the removed specimen -composed of spindle-shaped cells without dysplasia, atypia or pleomorphism- confirms the diagnosis of leiomyoma.

\section{DISCUSSION}

Leiomyoma of the bladder is a relatively rare tumor: less than $1 \%$ of the tumors of this organ (1). In a systematic review of the literature carried out by Silva-Ramos et al. (2) the characteristics of patients affected by this tumor were: $75.6 \%$ were women, the media age of presentation was 45.3 years, $50 \%$ of patients presented urinary voiding symptoms.

Ultrasound, CT and MRI are regularly used to diagnose this tumor. It was once proposed that MRI by itself could confirm this diagnosis, nonetheless and considering it cannot differentiate mesenchymal tumors from the more common transitional cell tumors (TCT) the histopathology study is always necessary to confirm $(3,4)$. Recent findings, nonetheless, indicate that the Apparent Diffusion Coefficient -obtained by diffusion-weighted magnetic resonance- values are significantly lower in malignant lesions (e.g. TCT) when compared to those obtained from benign tumors (i.e. mesenchymal tumors). The specificity claimed by this technique certainly deserves further empirical results and analysis.

The underlying cause of leiomyoma of the bladder is still unclear. No recurrences or ma- 
lignant degenerations of leiomyomas have been reported. Treatment of leiomyoma of the urinary bladder is mainly surgical. Surgical excision has excellent prognosis after complete resection and should always be offered.

We reported the case of a leiomyoma of the bladder, successfully removed by laparoscopy without compromising the bladder capacity.

\section{ACKNOWLEDGMENTS}

Sustainability Strategy 2013-2014 Reproduction Group

\section{ARTICLE INFO}

Int Braz J Urol. 2013; 39: 432-4

Submitted for publication:

June 28, 2012

Accepted after revision:

January 23, 2013

\section{REFERENCES}

1. Blasco Casares FJ, Sacristán Sanfelipe J, Ibarz Servio L, Batalla Cadira JL, Ruiz Marcellán FJ. Characteristics of bladder leiomyoma in our setting. Arch Esp Urol. 1995; 48: 987-90.

2. Silva-Ramos M, Massó P, Versos R, Soares J, Pimenta A. Leiomyoma of the bladder. Analysis of a collection of 90 cases. Actas Urol Esp. 2003; 27: 581-6.

3. Park JW, Jeong BC, Seo SI, Jeon SS, Kwon GY, Lee HM: Leiomyoma of the urinary bladder: a series of nine cases and review of the literature. Urology. 2010; 76: 1425-9.

4. Sundaram CP, Rawal A, Saltzman B: Characteristics of bladder leiomyoma as noted on magnetic resonance imaging. Urology. 1998; 52: 1142-3.
Correspondence address: Dr. Margarita Ortiz Carrera 51D No. 62 Medellín, 29, Colombia Fax: + 574 219-6476 E-mail: margaritamariaortizm@gmail.com 